\title{
Trehalose, an easy, safe and efficient cryoprotectant for the parasitic protozoan Trypanosoma brucei
}

Yan-Zi Wen ${ }^{a}$, Bi-Xiu Su ${ }^{a}$, Shu-Shen Lyu ${ }^{b}$, Geoff Hide ${ }^{c, d}$, Zhao-Rong Lun ${ }^{\mathrm{a}}$, De-Hua Lai ${ }^{\text {** }}$

a. Center for Parasitic Organisms, State Key Laboratory of Biocontrol, School of Life Sciences, Sun Yat-Sen University, Guangzhou 510275, P.R. China

b. School of Chemistry and Chemical Engineering, Sun Yat-Sen University, Guangzhou 510275, P.R. China

c. Ecosystems \& Environment Centre, School of Environment and Life Sciences, University of Salford, Salford M5 4WT, UK

d. Biomedical Research Centre, School of Environment and Life Sciences, University of Salford, Salford M5 4WT, UK

*Corresponding author: laidehua@mail.sysu.edu.cn 


\begin{abstract}
Trehalose, a non-permeating cryoprotective agent (CPA), has been documented as less toxic and highly efficient at cryopreserving different kinds of cells or organisms. In the present study, trehalose was evaluated for its application in cryopreservation of both Trypanosoma brucei procyclic and bloodstream form cells. The cryopreservation efficiency was determined by the motility of trypanosomes after thawing, as well as a subsequent recovery and infectivity assessment. The viability of trypanosomes from cultivation that were frozen in a serial concentrations of trehalose showed similar results to glycerol and DMSO. Nevertheless, trypanosomes cryopreserved in $0.2 \mathrm{M}$ trehalose showed the best growth characteristic during subsequent cultivation. In addition, CPA cocktails with trehalose and permeating CPA glycerol or DMSO were developed and evaluated. Interestingly, trypanosomes in host (mouse) blood cryopreserved in $0.4 \mathrm{M}$ trehalose $+5 \%$ glycerol showed higher infectivity than those preserved in trehalose/DMSO cocktails as well as individually. Further investigations showed that, in comparison with slow freezing at $-80^{\circ} \mathrm{C}$, flash freezing in liquid nitrogen provided better cryopreservation for bloodstream forms than slow freezing. In conclusion, trehalose is an easy, safe and efficient CPA for cryopreservation of $T$. brucei and potentially for other protozoan species and cells.
\end{abstract}

Key words: Trypanosoma brucei, trehalose, cryopreservation, cryoprotective agent 


\section{Introduction}

Trypanosoma brucei is a zoonotic parasitic protozoan distributed in Africa. Its subspecies are T. b. gambiense and T. $b$. rhodesiense which cause Africa sleeping sickness in humans, while the other subspecies T. b. brucei is a pathogen of domestic animals. To adapt to the mammalian and insect host, T. brucei undergoes cell differentiation through various life stages. The bloodstream form stage (BSF) in the mammalian host and the procyclic form stage (PCF) in the insect mid-gut are two well-studied stages in the laboratory. BSF and PCF from in vitro culture and BSF from mice can proliferate and provide sufficient amounts of parasites for investigation.

Genetic engineering techniques and field/medical sampling results in large numbers of collections of $T$. brucei clones/stains/isolates. To precisely preserve these samples, a common method is cryopreservation, which can significantly reduce the cost and inconvenience imposed by continual passage in animals or cultivation and it also limits the biological changes during passage. For cryopreservation, samples are usually frozen and stored below $-80^{\circ} \mathrm{C}$, typically in liquid nitrogen $\left(-196^{\circ} \mathrm{C}\right)$ (Karlsson et al., 1996). During the freezing phase, the water inside and outside of the cells forms crystals, which may mechanically damage the cell membrane and other cellular structures and lead to cell death after thawing (Fahy et al., 1984). Therefore, the key step for increasing the survival rate during the cryopreservation is the process of vitrificaton, that limits ice crystal formation and avoids crystalline damage, which is achieved by adding an appropriate antifreeze substance (a cryoprotective agent (CPA)) (Meryman, 1971).

To fulfill the need for cryopreservation, a variety of simple or complex chemical compounds have been developed as CPAs. One class of CPAs can freely permeate cellular membranes. For example, dimethylsulfoxide (DMSO or $\mathrm{Me}_{2} \mathrm{SO}$ ) is able to readily and rapidly diffuse across lipid bilayers and reduces water activity within cells which leads to effective protection against cell damage (Lovelock \& Bishop, 1959). While, another compound glycerol can penetrate the membrane and protect the cells 
by increasing viscosity which inhibits ice crystal growth before achieving the glassy matrix state (Fuller, 2004). The use of glycerol and DMSO, which are regarded as universally useful CPAs for most samples, actually marked the beginning of modern cryotechnology (Polge et al., 1949; Lovelock \& Bishop, 1959). The other class of CPAs comprises nonpermeating agents, such as hydroxyethyl starch, polyvinyl pyrrolidone and various sugars (Karlsson et al., 1996; McGann, 1978). Among them, trehalose has been used as a popular extracellular CPA in recent decades. It is a disaccharide of glucose and widely found in many kinds of organisms and is involved as an energy source, cell structural component and in metabolic pathways (Elbein, 1974; Elbein \& Mitchell, 1973). Early in 1964, a high concentration of trehalose was discovered in sawfly larva which can be resistant to temperatures as low as $-40^{\circ} \mathrm{C}$ (Asahina \& Tanno, 1964). Therefore, it was thought that trehalose might be able to participate in the process of maintaining life during freezing. Further study showed that the protection given by trehalose was attributed to its ability to form hydrogen bonds in bimolecular structures and thereby establishing a stable glassy matrix during cryopreservation (Clegg et al., 1982; Lawson et al., 2011). This not only can prevent the functional molecules from water deficit but also can protect the cell membrane from damage by ice crystallization. So far, trehalose has been documented as an efficient CPA in a wide variety of applications ranging from blood banks to reproductive technology (Lee et al., 2013; Limaye et al., 2001).

Trypanosoma spp. have been effectively cryopreserved in traditional CPAs for many years. Some of them have achieved better cryoprotective status in glycerol than in DMSO (Ndao et al., 2004), while others have displayed higher viability with DMSO (Walker \& Ashwood-Smith, 1961). However, the protocol for cryopreservation of T. brucei collected in the field is less convenient and shows impaired infectivity or even complete loss (Brun et al., 2001; Matovu et al., 2001). This protocol has been improved partially in recent decades. Maina et al. (2006) reported that they used commercial cryomedium Triladyl for T. brucei gambiense, which was the only application available for cryopreservation of bloodstream forms. Here, we have modified a protocol for cryopreservation of T. brucei, using an 
extracellular CPA trehalose based on our previous observation (Wang et al., 2014). We optimized the formula of trehalose as CPA and shortened the protocol during the cooling phase and subsequent cultivation after cryopreservation. Results of the current study suggest trehalose as an easy, safe and efficient CPA for cryopreservation of $T$. brucei, and may provide valuable data for the development of highly efficient CPAs for other single cells and/or even metazoans.

\section{Materials and Methods}

\subsection{Agents used for cryopreservation}

Trehalose (Sigma, USA) used at the concentration of $0.4 \mathrm{M}(\mathrm{mol} / \mathrm{L})$ provided the best protective effect for Trypanosoma brucei in blood as previously described (Wang et al., 2014). Hence, the CPA cocktails based on $0.4 \mathrm{M}$ trehalose with 5\% glycerol or $3.75 \%$ DMSO were tested on bloodstream stages from blood.

Trehalose at a series concentrations of $0.05 \mathrm{M}, 0.1 \mathrm{M}, 0.2 \mathrm{M}, 0.3 \mathrm{M}, 0.4 \mathrm{M}, 0.5 \mathrm{M}$ were tested on PCF and BSF cells cultivated in vitro. For these cells, CPA cocktails were made from trehalose at the concentration offering the best cryopreservation $(0.2$ M) mixed with 5\% and 10\% glycerol, or 3.75\% and 7.5\% DMSO.

Widely and commonly used CPAs for trypanosomes, include $10 \%$ glycerol and 7.5\% DMSO and so thesewere used as the controls. All mentioned CPA formulas are given as a final concentration and working aliquots were prepared at a two times $(2 \mathrm{x})$ concentration for an easy mix at a volume ratio of 1:1 with trypanosome suspensions. All CPAs for BSF in blood were dissolved or diluted with PBS buffer (pH 7.4). All CPAs for PCF or BSF cells from in vitro culture were dissolved or diluted with either complete SDM-79 medium or complete HMI-9 medium, respectively.

\subsection{Trypanosomes}

The T. brucei STIB 920 strain was thawed from liquid nitrogen and injected into mice. Mice were treated in strict accordance to the guidelines for Medical Laboratory Animals (1998) from Ministry of Health, China, under the protocols approved by National Institute for Communicable Disease Control and Prevention and Laboratory 
Animal Use and Care Committee of Sun Yat-Sen University under the license 2010CB53000. The bloods of mice were collected from heart when the parasitemia reached $10^{8} / \mathrm{ml}$ and prepared for cryopreservation.

T. brucei PCF cell line $29-13$ was maintained at $27^{\circ} \mathrm{C}$ and $\mathrm{BSF}$ cell line Antat 1.1 was maintained at $37^{\circ} \mathrm{C}$ and both were supplied with humidified air containing $5 \%$ $\mathrm{CO}_{2}$. The cells at logarithmic growth phase (PCF, about $10^{7} / \mathrm{ml}$; BSF, about $10^{6} / \mathrm{ml}$ ) were used for cryopreservation.

\subsection{Cryopreservation of trypanosomes}

Trypanosomes cultivated in vitro or in blood collected from infected animals were suspended well before adding given CPAs in a dropwise manner to a volume ratio of $1: 1$. Then, the mixture $(200 \mu \mathrm{l}$ aliquot) was loaded into a $1 \mathrm{ml}$ cryotube (Thermo Fischer Scientific, Denmark). In flash freezing protocols, the tubes were directly plunged into liquid nitrogen $\left(-196^{\circ} \mathrm{C}\right)$; while in slow freezing protocols, the tubes were cooled at $-1{ }^{\circ} \mathrm{C} / \mathrm{min}$ by placing in a cell freezing container (ExCell Bio, China), placed in a freezer at $-80^{\circ} \mathrm{C}$ for more than 2 hours and later immersed in liquid nitrogen.

After storage for more than 48 hours in liquid nitrogen, trypanosomes were completely thawed in a water bath at $27^{\circ} \mathrm{C}(\mathrm{PCF})$ or $37^{\circ} \mathrm{C}(\mathrm{BSF})$ for about 30 seconds, and rapidly evaluated for the following parameters: morphology, mobility and quantity.

\subsection{Cell number counts}

Trypanosomes in infected blood were diluted 200 times with PBS buffer and only motile ones were counted by microscopy using a haemocytometer (HBG, Germany).. Trypanosomes in culture media were counted by using a CASY counter (Roche, USA). The survival percentage was calculated by comparison with the original numbers of trypanosomes prior to cryopreservation.

2.5 Recovery and infectivity of trypanosomes after cryopreservation. 
Most cryopreservation studies have demonstrated good survival rates (motility) of parasitic parasites, but the original biological and metabolic characteristics after cryopreservation which are strongly linked to the efficiency of propagation have been rarely described. Here, not only the post-thaw motility, but also the recovery ability and the infectivity of trypanosomes were evaluated as described below. For in vitro cultivation, PCF cells and BSF cells were diluted upon thawing to $10^{6} / \mathrm{ml}$ and $10^{5} / \mathrm{ml}$ (5-fold dilutions) using corresponding media, respectively. These were seeded into 24-well culture plate and examined daily by microscopy. The growth of cells was recorded after 48 hours.

BSF cells $\left(10^{5}\right)$ in blood were injected to mice after thawing using six replicates per group. Tail blood from each mouse was checked and the parasitemia was recorded every 12 hours for three days.

\subsection{Statistical analysis}

Statistic analyses were performed using SPSS statistical soft-ware 12.0 (SPSS Inc, Chicago). The survival rate, the quantity of cells in vitro and the parasitemia in each group were analyzed by independent sample $t$-test. For all analyses, $\mathrm{P}<0.05$ was considered significant.

\section{Results}

\subsection{Optimum trehalose concentration for trypanosomes in vitro}

Although our previous study demonstrated that $0.4 \mathrm{M}$ trehalose could provide the best cryoprotective effect to Trypanosoma brucei in host blood (Wang et al., 2014), it has never been tested using trypanosomes collected from cultivation in vitro. Here, we applied trehalose at a concentration ranging from $0.05 \mathrm{M}$ to $0.5 \mathrm{M}$ for the cryopreservation of T. brucei in vitro. Interestingly, all PCF and BSF cells frozen in the experimental concentrations of trehalose displayed high survival capacity (more than 90\%) following thawing and were as good as the ones using glycerol and DMSO (Fig.S1 A, B). Further, in order to test the viability, trypanosomes were directly diluted with the respective culture medium with a 5-fold dilutionfor cultivation following thawing. Results from the recovery experiments demonstrated that $0.2 \mathrm{M}$ 
trehalose provided dramatically higher viability after 48 hours incubation (Fig.1 A, B) compared to other concentrations of trehalose $(\mathrm{P}<0.05$ to $\mathrm{P}<0.00005)$ and DMSO or Glycerol $(\mathrm{P}<0.005$ or $\mathrm{P}<0.00005)$.

Since the remaining CPA in the culture medium may be toxic to trypanosomes during the incubation, we tested the toxicity of trehalose during cell cultivation by incubation of both BSF cells and PCF cells with serial dilutions of trehalose (0-0.2 M). There was no significant difference between the groups with trehalose (0.05 $\mathrm{M}$ or less) and the control (0 M) (Fig. S2). This indicates that trehalose at the concentration of $0.05 \mathrm{M}$ or less does not inhibit the growth of both PCF cells and BSF cells $(\mathrm{P}<0.005$ to $\mathrm{p}<0.00005)$. Therefore, in our studies, trehalose at the concentration of $0.2 \mathrm{M}$ for cryopreservation followed by a 5-fold dilution (final trehalose at $0.04 \mathrm{M}$ ) or more, will be free from CPA toxicity during subsequent cultivation. This demonstrates that this is the optimum concentration for preserving trypanosomes in vitro.

\subsection{The use of cocktails of CPAs for trypanosome cryopreservation in vitro}

In order to avoid possible cytotoxic effects, CPA cocktails were introduced to decrease the concentration necessary to achieve successful vitrification. Here, we tested potential CPA cocktails with a combination of extra-cellular (trehalose) and intra-cellular agents (glycerol or DMSO) for trypanosomes in vitro.

Trypanosomes that were preserved in either individual compounds or in cocktails of CPAs displayed a high survival capacity after cryopreservation (>90\%) (Fig. S1C, D). However, after dilution with fresh medium and initiation of first $48 \mathrm{hr}$ cultivation, the trypanosomes frozen in the cocktails displayed growth superiority to all those in the cocktails $(\mathrm{P}<0.05$ to $\mathrm{P}<0.000005)$ (Fig. $1 \mathrm{C}, \mathrm{D})$.

\subsection{Optimal cryoprotectants for trypanosomes in blood}

Although $0.4 \mathrm{M}$ trehalose provided the highest survival capacity of T. brucei in frozen blood of as reported in our previous studies (Wang et al., 2014), we also evaluated the cryoprotective effect of CPA cocktails for trypanosomes in blood. The motility of trypanosomes in blood was almost the same after cryopreservation in all CPAs and 
CPA cocktails (P> 0.05) (Fig. S1E). Furthermore, the infectivity capacity was investigated by direct injection (i.p.) of thawed cells into the mice. Predictably, trypanosomes from all CPA groups remained infectious to the mice (Fig. 1E). However, compared to the other agents, the parasitaemia in the mice infected with trypanosomes frozen with $0.4 \mathrm{M}$ trehalose $+5 \%$ glycerol was higher than other CPA groups at 72 hours after injection $(\mathrm{P}<0.05$ to $\mathrm{P}<0.00005)$ (Fig. 1E). This indicated that a cocktail of both intra- and extra-cellular preserving agents may actually provide a better level of cryopreservation in bloodstream forms.

\subsection{Flash freezing provides better protection than slow freezing for trypanosomes}

The prior cooling rate during cryopreservation is a critical parameter in avoiding ice crystallization (Sutton, 1991 \& 1992; Peyridieu et al., 1996). Commonly, in the laboratory, cell freezing containers have been widely used. These cool cells slowly at a rate of approximately $-1{ }^{\circ} \mathrm{C} / \mathrm{min}$ in a $-80^{\circ} \mathrm{C}$ freezer. It has generally been considered that during the cell cryopreservation, slow freezing better protects cells from damage (Dalgliesh, 1972). Flash freezing, another commonly used protocol, involving immersion of cells in liquid nitrogen seems more convenient.

Here, we tested the effect of a combination of CPAs in cryopreservation of $T$. bruce $i$ with flash freezing compared to slow freezing. Trypanosomes were randomly divided into two groups after being mixed with CPAs. One group was frozen with the temperature falling to $-80^{\circ} \mathrm{C}$ gradually and then transferred into the liquid nitrogen. The other group was directly immersed into the liquid nitrogen. The survival percentages of $\mathrm{BSF}$ in vitro treated with both distinct methods were the same $(\mathrm{P}>0.05)$ (Fig. S1G). Interestingly, the vitality of those flash frozen in liquid nitrogen appeared significantly stronger than those treated with slowly freezing method after cultivation $(\mathrm{P}<0.05$ to $\mathrm{P}<0.00005)$ (Fig. 2B). Moreover, higher survival ratios of $\mathrm{BSF}$ trypanosomes in blood were also observed in the group treated with flash freezing $(\mathrm{P}<0.05$ to $\mathrm{P}<0.005)$ (Fig. 2C). These indicated that flash freezing could provide more effective protection for BSF cells that were both in cultivated in vitro and in vivo. While in the case of PCF cells, indistinctive results were observed from both freezing 
methods with CPAs of either trehalose or glycerol (P>0.05) (Fig S2A, S1F).

\section{Discussion}

The conventional methods for preservation of parasites by continual passage are limited by the initial isolation and loss of strains, bacterial and fungal contamination, and changes in the original biological and metabolic characteristics (Miyake et al., 2003). These shortages are considerably reduced when the samples are cryopreserved. For several decades, a key mechanism involved in the cryopreservation of cells or organisms has been recognized as vitrification, a transformation from liquid to an amorphous state without any crystallization (Fahy et al., 1984). To this goal, various kinds of compounds have been tested for their efficiencies as cryoprotectant agents. CPAs could promote the vitrification and provide protection for the cells from cryoinjury, which is usually caused by the formation of ice crystal during freezing and thawing. Ice-free cryopreservation can be achieved only when the cells or organisms are loaded with high concentrations of CPA. However, this is a double-edged sword, as it comes with the side effect of high toxicity potential caused by excessive CPA (Fahy, 1986). Therefore, the toxicity of CPA to the cells or organism during cryopreservation cannot be ignored. For example, glycerol molecules may insert into the lipid bilayer, alter the stability of the membrane and influence the cell viability or even lead to death (Leibo et al., 1978). It has been reported that, the present of even a very low concentration of glycerol $(4 \mathrm{mM})$ in the medium, can partially inhibit the growth of trypanosomes (Lai et al., 2014). DMSO has been shown to alter gene expression and induce genome wide changes in gene activity (Iwatani et al., 2006; Pal et al., 2012). It can also favor protein aggregation which affects protein content and function (Giugliarelli et al, 2016). In fact, cytotoxicity was observed in Trypanosoma congolense where infectivity was markedly reduced if exposed to DMSO (Overdulve et al., 1970). Consequently, in general, a centrifugation step is usually performed to discard the CPAs after cryopreservation. Then cells are then resuspended in fresh culture medium before starting a new round of culture. 
Here, we have shown that trypanosomes could be co-cultured with trehalose at a concentration lower than $0.05 \mathrm{M}$ without any growth inhibition (Fig. S2). In fact, in our hands, the best recovery results were observed in the trypanosomes which were frozen with $0.2 \mathrm{M}$ trehalose. An easy dilution of thawed cells at about 5 times or more is sufficient to avoid the toxicity of trehalose in the subsequent cultivation. Therefore, no centrifugation or other steps are required and simplifies the post-thawing protocol this saving time. In addition, this observation also suggests that, in comparison to glycerol and DMSO, trehalose is a better CPA with much lower cytotoxicity. Actually, trypanosomatids (in some life cycle stages atleast) parasitize insects, an environment rich of trehalose (Wyatt and Kale, 1957). Hence, the presence of trehalose phosphorylase in their genomes indicates trehalose may be metabolized into glucose and glucose 6-phosphate (as summarized in Oppedoes et al., 2016), and therefore, a potential carbon source in these organisms.

However, it is well documented that CPAs may damage the cells if present in excess. Over dose of trehalose may lead to over dehydration resulting in cell death (Fowler \& Toner, 2005). We previously found that at concentrations of more than 0.4 M of trehalose, T. brucei motility was significantly impaired (Wang et al., 2014). In order to avoid such damage, a combination strategy using two or more CPAs, known as CPA cocktails, could help to reduce the required amount of each corresponding CPA. Malo and colleagues reported that the addition of trehalose to glycerol could provide better protection for the spermatozoa against freeze damage (Malo, et al., 2010). Our results also demonstrated that the combination of trehalose and glycerol could also provide better protection for bloodstream T. brucei than trehalose or glycerol alone, because, the parasitemia of mice injected with bloodstream trypanosomes frozen in $0.4 \mathrm{M}$ trehalose $+5 \%$ glycerol apparently increased faster than trypanosomes frozen in CPAs alone. We think that glycerol may be a valuable partner for avoiding cryoinjury compared with trehalose alone. However, it was in contrast to the results obtained for the cultured trypanosomes, which might be due to the neutralization of glycerol toxicity by the presence of a high concentration of serum/blood. The attempt use of DMSO as a CPA partner showed no improvement 
for trypanosomes in blood, which may due to incompatibility and the combinations of DMSO with other agents probably increases toxic potential (Brayton, 1986).

In general, besides the type and concentration of CPA, the cooling rate is regarded as crucial to effective cryopreservation (Sutton, 1991; Peyridieu, et al., 1996). The control of cooling rate at a descrease of $1^{\circ} \mathrm{C} / \mathrm{min}$ is routinely used for preserving parasitic protozoa (Dalgliesh, 1972) although, some studies suggest that different organisms required different cooling rates (Miyake et al., 2003). Interestingly, results from our current works in comparison of the effects of flash and slow freezing on trypanosomes, showed that trypanosomes in the blood displayed a higher survival ratio in flash frozen irrespective of which CPA was used. Interestingly, BSF cells in vitro which were treated using flash freezing showed growth advantages. As a matter of fact, a similar phenomenon has been described in Trypanosoma cruzi (Filardi \& Brener, 1975). These results might be explained by a longer exposure time to the CPA during slow cooling resulting in higher toxicity due to increased ice crystal formation. Therefore we conclude, based on comparisons with the use of the freezing box, that direct immersion of BSF into liquid nitrogen is not only much more convenient but also efficient. Miyake and colleagues demonstrated that T. brucei gambiense could be cryopreserved over a wide range of cooling rates (Miyake et al., 2003). We also found that there was no significant difference between the PCF when flash or slow frozen in trehalose and glycerol, other than when carried out in DMSO. It is possible that the PCF may be less sensitive to ice crystal formation and therefore experience less toxicity with glycerol and trehalose than DMSO.

So far, except for trypanosomes, most protozoan parasites such as Toxoplasma gondii, Giardia lamblia, Trichomonas, Leishmania have been reported successfully cryopreserved with various CPAs. However, most of these cells were preserved with glycerol or DMSO (Reviewed by Hubalek, 2003). Actually, a simple try on Leishmania cryopreservation in our lab found that $0.4 \mathrm{M}$ trehalose work as well as $10 \%$ glycerol. Based on our results from trehalose or trehalose based cocktails, which provided a better cryopreservation experience than glycerol and DMSO, we suggest that trehalose or trehalose cocktails could be used for cryopreservation in other 
parasitic protozoan and animal cells.

\section{Acknowledgments}

AcknowledgmentsWe would like to thank the technique support from BSc Cong-XiZhang. The work was supported by the grants from the National Sciences

Foundation of China (\#31272305, \#31472058, \#31301876, \#31402029) and Fundamental Research Funds for the Central Universities (\#141gpy23). 


\section{References}

Asahina, E., Tanno, K.S., 1964. A large amount o trehalose in a frost-resistant insect. Nature 204, 1222.

Brayton, C.F., 1986. Dimethyl sulfoxide (DMSO): a review. Comell. Vet. 76, 61-90.

Brun, R., Schumacher, R., Schmid, C., Kunz, C., Burri, C., 2001. The phenomenon of treatment failures in human African trypanosomosis. Trop. Med. Int. Health 6, 906-914.

Clegg, J.S., Seitz, P., Seitz, W., Hazlewood, C.F., 1982. Cellular responses to extreme water loss: the water-replacement hypothesis. Cryobiology 19, 306-316.

Dalgliesh, R.J., 1972. Theoretical and practical aspects of freezing parasitic protozoa. Aust. Vet. J. 48, 233-239.

Elbein, A.D., 1974. The metabolism of $\alpha, \alpha$-trehalose. In :Tipson, R. S., Horton, D. (Eds.), Advances in Carbohydrate Chemistry and Bio-chemistry, vol. 30. Academic Press, New York, pp. 227-256.

Elbein, A.D., Mitchell, M., 1973. Levels of glycogen fand trehalose in Mycobacterium smegmatis and the purification and properties of the glycogen synthetase. J. Bacteriol. 863-873.

Fahy, G.M., MacFarlane, D.R., Angell, C.A., Meyman, H.T., 1984.Vitrification: An approach to cryopreservation. Cryobiology 21, 407-426.

Fahy, G.M., 1986. The revelance of cryoprotectant "toxicity" to cryobiology. Cryobiology 23, 1-13.

Filardi, L.S., Brener, Z., 1998. Cryopreservation of Trypanosoma cruzi bloodstream forms. J. Protozool. 22, 398-401.

Fowler, A., Toner, M., 2005. Cryo-injury and biopreservation. Ann. NY. Acad. Sci. 1066, 119-135.

Fuller, B.J., 2004. Cryoprotectants: the essential antifreezes to protect life in the frozen state. Cryo-letter. 56, 265-270.

Giugliarelli, A., Urbanelli, L., Ricci, M., Paolantoni, M., Emiliani, C., Saccardi, R., Mazzanti, B., Lombardini, L., Morresi, A., Sassi, P., 2016. Evidence of 
DMSO-induced protein aggregation in cells. J. Phys. Chem. A.

Hubalek, Z., 2003. Protectants used in the cryopreservation of microorganisms. Cryobiology 46, 205-229.

Iwatani, M., Ikegami, K., Kremenska, Y., Hattori, N., Tanaka, S., Yagi, S., Shiota, K., 2006. Dimethyl sulfoxide has an impact on epigenetic profile in mouse embryoid body. Stem Cells 24, 2549-2556.

Karlsson, J.O.M., Toner, M., 1996. Long-term storage of tissues by cryopreservation: critical issues. Biomaterials. 17, 243-256.

Lai, D.H., Poropat, E., Pravia, C., Landoni, M., Couto, S.A., Rojo, P.G.F., Fuchs, G.A., Dubin, M., Elingold, I., Rodriguez, B.J., Ferella, M., Esteva, I.M., Bontempi, J.E., Lukes, J., 2014. Solanesyl diphosphate synthase, an enzyme of the ubiquinone synthetic pathway, is required throughout the life cycle of Trypanosoma brucei. Eukaryotic Cell 13, 320-328.

Lawson, A., Ahmad, H., Sambanis, A., 2011. Cryotoxicty effects of cryoprotectants as single-component and cocktail vitrificaiton solutions. Cryobiology 62, 115-122.

Lee, Y.A., Kim, Y.H., Kim, B.J., Kim, B.G., Kim, K.J., Auh, J.H., Schmidt, J.A., Ryu, B.Y., 2013. Cryopreservation in trehalose preserves functional capacity of murine spermatogonial stem cells. Plos One 8, e54889.

Leibo, S.P., McGrath, J.J., Cravalho, E.G., 1978. Microscopic observation of intracellular ice formation in unfertilized mouse ova as a function of cooling rate. Cryobiology 15, 257-271.

Limaye, L.S., Kale, V.P., 2001. Cryopreservation of human hematopoietic cells with membrane stabilizers and bioantioxidants as additives in the conventional freezing medium. J. Hematother Stem. Cell Res. 10, 709-718.

Lovelock, J.E., Bishop, M.W.H., 1959. Prevention of freezing damage to living cells by dimethylsulphoxide. Nature 183, 1394-1395.

Malo, C., Gil, L., Gonzalez, N., Cano, R., de, Blas, I., Espinosa, E., 2010. Comparing sugar type supplementation for cryopreservation of boar semen in egg yolk based extender. Cryobiology 61, 17-21.

Maina, N.W., Kunz, C., Brun, R., 2006. Cryopreservation of Trypanosoma brucei gambiense in a commercial cryomedium developed for bull semen. Acta Trop. 98, 207-211. 
Matovu, E., Enyaru, J.C.K., Legros, D., Schmid, C., Seebeck, T., Kaminky, R., 2001. Melarsoprol refractory T. b. gambiense isolates from Omugo north-western Uganda. Trop. Med. Int. Health 6, 407-411.

McGann, L.E., 1978. Differing actions of penetrating and nonpenetrating cry protective agents. Cryobiology 15, 382-390.

Meryman, H.T., 1971. Cryoprotective agents. Cryobiology 8, 173-183.

Miyake, Y., Karanis, P., Uga, S., 2003. Cryopreservation of protozoan parasites. Cryobiology 48, 1-7.

Ndao, M., Magnus, E., Büscher, P., Geerts, S., 2004. Trypanosoma vivax: a simplified protocol for in vivo growth, isolation and cryopreservation. Parasite. 11, 103-106.

Oppedoes, F.R., Butenko, A., Flegontov, P., Yurchenko, V., Lukes, J., 2016.

Comparative Metabolism of free-living Bodo saltans and parasitic Trypanosomatids. J. Eukaryot. Microbiol. 63, 657-678.

Overdulve, J.P., Antonisse, H.W., 1970. Measurement of the effect of low temperature on protozoa by titration. II. Titration of Babesia rodhaini, using prepatent period and survival time, before and after strorage at minus 76 degree C. Exp. Parasitol. 27, 323-341.

Pal, R., Mamidi, M.K., Das, A.K., Bhonde, R., 2012. Diverse effects of dimethyl sulfoxide (DMSO) on the differentiation potential of human embryonic stem cells. Arch. Toxicol. 86, 651-661.

Peyridieu, J.F., Baudot, A., Boutron, P., Mazuer, J., Odin, J., Ray, A., Chapelier, E., Payen, E., Descotes, L., 1996. Critical cooling and warming rates to avoid ice crystallization in small pieces of mammalian organs permeated with cryoprotective agents. Cryobiology 33, 436-446.

Polge, C., Smith, A.U., Parkes, A.S., 1949. Revival of spermatozoa after vitrification and dehydration at low temperatures. Nature 164, 666.

Sutton, R.L., 1991. Critical cooling rates to avoid ice crystallization in solutions of cryoprotective agents. J. Chem. Soc. 87, 101-105.

Sutton, R.L., 1992. Critical cooling rates for aqueous cryoprotectants in the presence of sugars and polysaccharides. Cryobiology 29, 585-598.

Walker, P.J., Ashwood-Smith, M.J., 1961. Dimethylsulphoxide, an alternative to glycerol, for the low-temperature preservation of trypanosomes. Ann. Trop. Med. 
Parasitol. 55, 93-96.

Wang, H.Y., Wen, Y.Z., Lun, Z.R., Lu, S.S., 2014. Visual observation of African trypanosomes during cryopreservation. Biopreserv. Biobank. 12, 265-268.

Wyatt, G.R., Kale, G.F., 1957. The chemistry of insect hemolymph. II. Trehalose and other carbohydrates. J. Gen. Physiol. 40, 833-847. 


\section{Figure legends}

Fig. 1 The optimal CPAs for cryopreserving trypanosomes. (A, B, C, D) the quantity of cells following cultivation in vitro for $48 \mathrm{~h}$ after cryopreservation, $n=3$. (E) the parasitemia in mice after injection with trypanosomes after cryopreservation post $72 \mathrm{~h}$, $\mathrm{n}=6$. Statistical significance levels for the $0.2 \mathrm{M}$ trehalose group (A, B, C, D) or 0.4 $\mathrm{M}$ trehalose $+5 \%$ glycerol cocktail (E) group compared to other CPA groups are indicated by asterisks: *, $\mathrm{p}<0.05 ; * *, \mathrm{p}<0.005 ; * * *, \mathrm{p}<0.0005 ; * * * *, \mathrm{p}<0.00005$; *****, $\mathrm{p}<0.000005 . \mathrm{G}$, glycerol; D, DMSO; T, trehalose..

Fig. 2 The cryoprotective effect on Trypanosoma brucei when applying flash freezing (liquid nitrogen) and slow freezing $\left(-80^{\circ} \mathrm{C}\right)$ protocols. The recovery of procyclic form cells (A) and bloodstream form cells (B) from cryopreservation after $48 \mathrm{~h}$ cultivation in vitro. The survival ratios of bloodstream form in blood after cryopreservation (C).Statistically significant differences are indicated by asterisks: *, p $<0.05$; **, p < $0.005, * * * *, \mathrm{p}<0.00005, \mathrm{n}=3$. G, glycerol; D, DMSO; T, trehalose. 
Fig. 1

1A

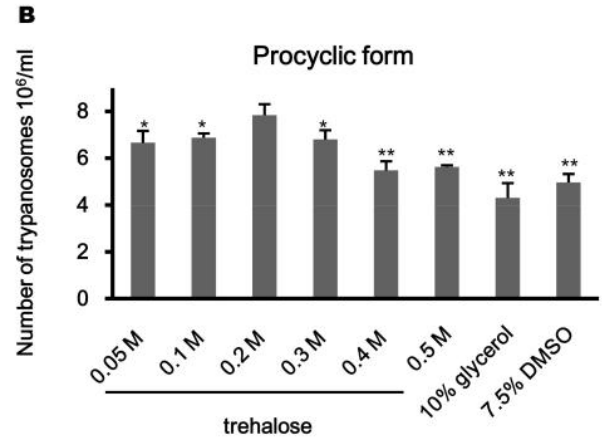

$1 \mathrm{C}$

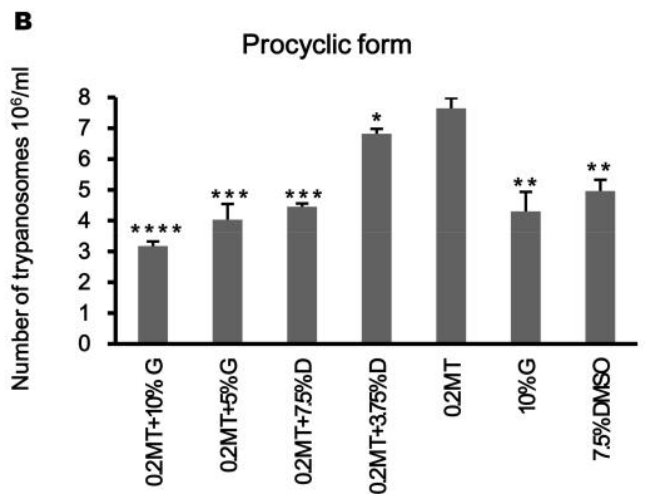

$1 \mathrm{E}$

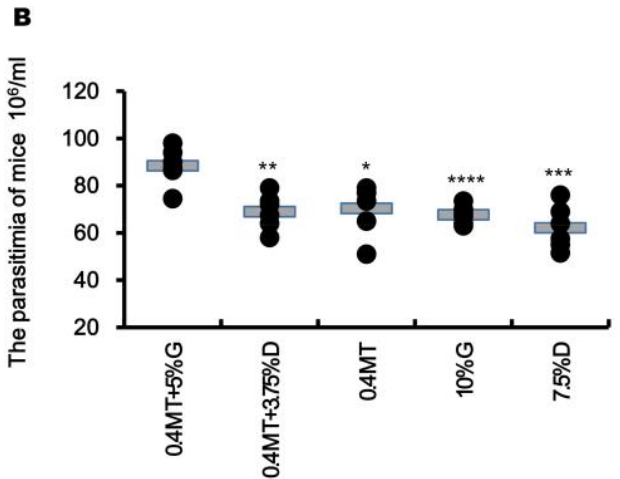

1B

D

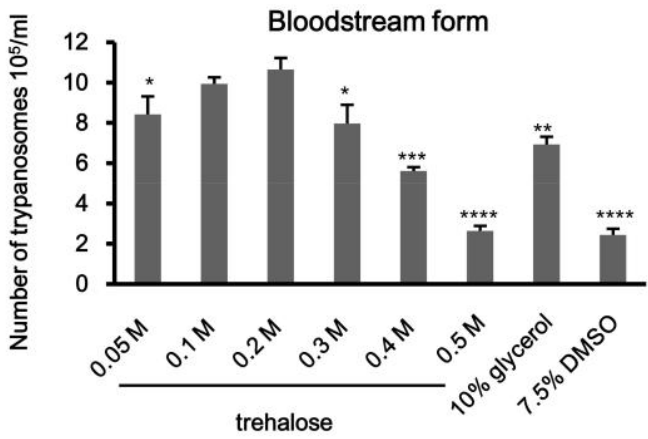

1D

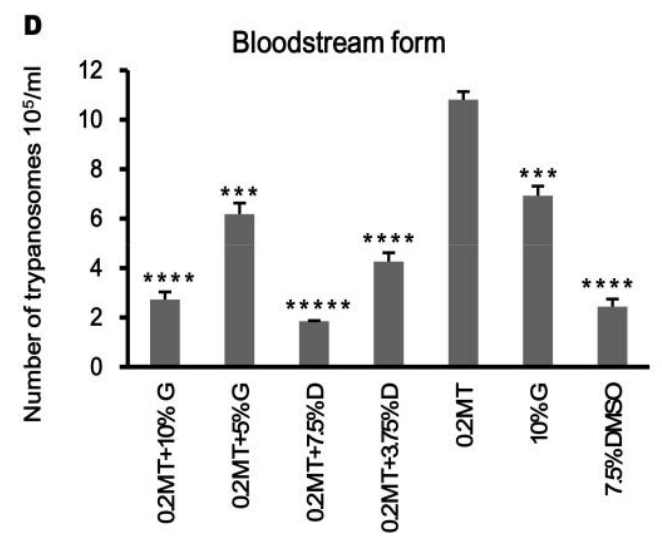


FIG 2

2A

B

Procyclic form

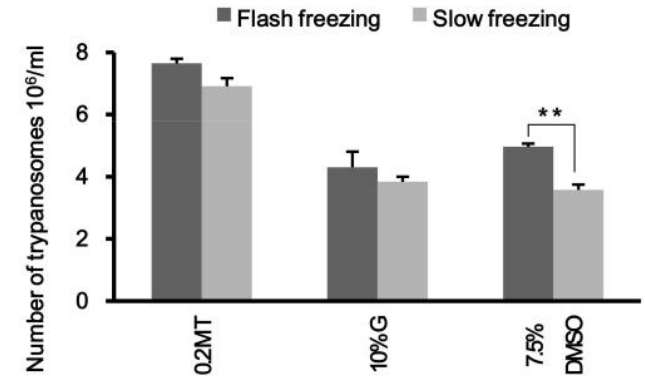

2C

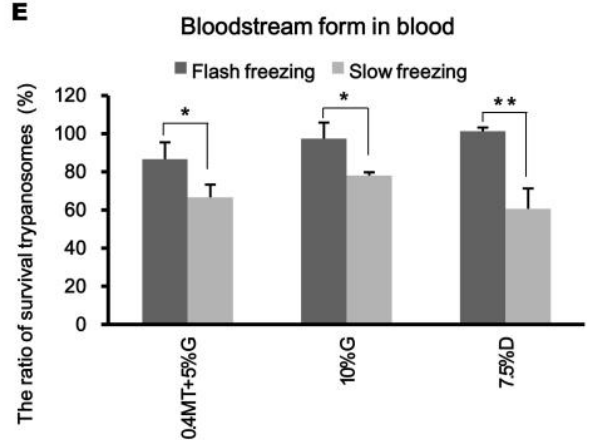

2B

D

Bloodstream form

- Flash freezing $\quad$ Slow freezing

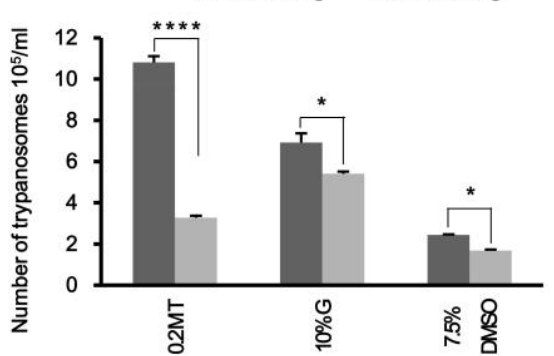


